Pacific

Journal of

Mathematics

\title{
NONLINEAR EULER SUMS
}

ISTVÁN MEZŐ

Volume 272 No. 1

November 2014 


\title{
NONLINEAR EULER SUMS
}

\author{
ISTVÁN MEZŐ
}

\begin{abstract}
We work out some formulas for nonlinear Euler sums involving multiple zeta values. As applications of these formulas, we give new closed form sums of several nonlinear Euler series, we present sums for powers of the digamma function and deduce the Landen identities for the polylogarithms by finite combinatorial identities.
\end{abstract}

2. Existing results and research directions 203

3. New nonlinear Euler sum formulas 205

4. Generating functions of nonlinear Euler sums 215

5. The Landen functional equations of the dilogarithm and trilogarithm functions

6. Collected sums

Acknowledgement

\section{Introduction}

In a letter from Goldbach to Euler, Goldbach proposed to investigate infinite series of the form

$$
\sum_{n=1}^{\infty} \frac{1}{n^{a}} \sum_{k=1}^{n} \frac{1}{k^{b}} .
$$

See for the historical details. In 1742 and 1743 Euler presented a number of closed form expression for such sums and their variations. The most fundamental one is the following [Borwein and Bradley 2006]:

$$
\sum_{n=1}^{\infty} \frac{1}{n^{2}} \sum_{k=1}^{n-1} \frac{1}{k}=\sum_{n=1}^{\infty} \frac{1}{n^{3}}=8 \sum_{n=1}^{\infty} \frac{(-1)^{n}}{n^{2}} \sum_{k=1}^{n-1} \frac{1}{k} .
$$

The research of István Mező was supported by the Scientific Research Foundation of Nanjing University of Information Science and Technology.

MSC2010: 33B15, 11M32.

Keywords: Euler sums, nonlinear Euler sums, polygamma functions, polylogarithm functions,

Landen's identities, multiple zeta values. 
The sum in the middle is a zeta function value and in the present day we consider the value of this series as a "fundamental constant", which cannot be traced back to "more fundamental" ones.

In the past two hundred years it had been apparent that the above sums and their generalizations - nowadays they are called Euler sums — often can be traced back to zeta function values. To treat these sums, we adopt the modern notations and notions. The multiple polylogarithm is defined by

$$
\zeta\left(s_{1}, s_{2}, \ldots, s_{m} ; z\right)=\sum_{0<n_{m}<\cdots<n_{1}} \frac{z^{n_{1}}}{n_{1}^{s_{1}} n_{2}^{s_{2}} \cdots n_{m}^{s_{m}}},
$$

with the appropriate restriction on the powers to get a convergent series. In particular,

$$
\zeta(s ; 1)=\zeta(s)=\sum_{n=1}^{\infty} \frac{1}{n^{s}} \quad(\Re(s)>1)
$$

is the classical Riemann zeta function [Andrews et al. 1999]. Typically $z$ is set to 1 or -1 , in which cases we are dealing with a multiple zeta function or alternating multiple zeta function, respectively. We remark that in the literature there exists a more general version of the above multiple zeta function, called the colored multiple zeta function [Bigotte et al. 2002]. It is defined as

$$
\zeta\left(s_{1}, s_{2}, \ldots, s_{m} ; \sigma_{1}, \sigma_{2}, \ldots, \sigma_{m}\right)=\sum_{0<n_{m}<\cdots<n_{1}} \frac{\sigma_{1}^{n_{1}} \sigma_{2}^{n_{2}} \cdots \sigma_{m}^{n_{m}}}{n_{1}^{s_{1}} n_{2}^{s_{2}} \cdots n_{m}^{s_{m}}} .
$$

The sum

$$
\sum_{i=1}^{m} s_{i}
$$

is the weight of the zeta function, while $m$ is the depth. A brief survey on multiple polylogarithms can be found in [Bowman and Bradley 2001].

The finite sums inside the sums are called generalized harmonic numbers and are denoted by $H_{n, r}$ (or $H_{n}^{(r)}$, but we use the former, because our expressions will involve powers):

$$
H_{n, r}=\sum_{k=1}^{n} \frac{1}{k^{r}} \quad(n \geq 1, r \geq 1),
$$

with the convention $H_{0, r}=0$ for all $r=1,2, \ldots$ The numbers $H_{n, 1}=: H_{n}$ are called harmonic numbers.

With these, the above relations under (1) can be written in the short form

$$
\zeta(2,1):=\sum_{n=1}^{\infty} \frac{H_{n-1}}{n^{2}}=\zeta(3)=8 \zeta(2,1 ;-1) .
$$


For Euler's original proof, see [Euler 1776]. These relations were rediscovered many times, as the references [Briggs et al. 1955; Bruckman 1982; Farnum and Tissier 1999; Klamkin and Steinberg 1952] show.

We mention that the general expression in terms of zeta values of the sum

$$
\sum_{n=1}^{\infty} \frac{H_{n}}{n^{a}}
$$

was known already to Euler, who found that

$$
\sum_{n=1}^{\infty} \frac{H_{n}}{n^{a}}=\left(1+\frac{a}{2}\right) \zeta(a+1)-\frac{1}{2} \sum_{k=1}^{a-2} \zeta(k+1) \zeta(a-k) \quad(a \geq 2) .
$$

Naturally, then, researchers after Euler have turned to generalizations and alterations of these sums. In the next section we present some existing directions, then we show in which direction we proceed.

\section{Existing results and research directions}

2.1. Alternating Euler sums. In the past and present, the alternating Euler sums and their modifications and generalizations have attracted the attention of a large number of mathematicians. For example, the alternating Euler sums, like

$$
\sum_{n=1}^{\infty} \frac{1}{n^{a}} \sum_{k=1}^{n} \frac{(-1)^{k}}{k^{r}}
$$

are investigated in [Bailey et al. 1994; de Doelder 1991; Li 2011; Sitaramachandra Rao 1987], to name a few. We mention one sum from [Li 2011, Proposition 3.2]:

$$
\sum_{n=1}^{\infty} \frac{1}{n^{2}} \sum_{k=1}^{n} \frac{(-1)^{k+1}}{k}=\frac{3}{2} \zeta(2) \log 2-\zeta(3) .
$$

In [Sitaramachandra Rao 1987] one can find an exhaustive bibliography on alternating Euler sums. It turns out that these sums are reducible to zeta values in many cases, see [Flajolet and Salvy 1998, Theorem 7.1].

2.2. Analytic extension of Euler sums. T. Apostol and T. H. Vu [1984] started to investigate Euler sums as functions of the power of $n$ :

$$
h(s)=\sum_{n=1}^{\infty} \frac{H_{n}}{n^{s}} .
$$

They showed that this function can be continued to the whole $s$-plane as a meromorphic function with a second-order pole at $s=1$, and simple poles at $s=0$ and at the negative odd integers. 
In the same paper this result was extended to the function

$$
h(s, z)=\sum_{n=1}^{\infty} \frac{H_{n, z}}{n^{s}}
$$

These results were further specified and extended by Boyadzhiev [2008; 2009] to

$$
\begin{aligned}
& u(s)=\sum_{n=1}^{\infty} \frac{(-1)^{n+1}}{n^{s}} H_{n}, \\
& v(s)=\sum_{n=1}^{\infty} \frac{(-1)^{n+1}}{n^{s}}\left(1-\frac{1}{2}+\frac{1}{3}+\cdots+\frac{(-1)^{n+1}}{n}\right), \\
& w(s)=\sum_{n=1}^{\infty} \frac{1}{n^{s}}\left(1-\frac{1}{2}+\frac{1}{3}+\cdots+\frac{(-1)^{n+1}}{n}\right) .
\end{aligned}
$$

2.3. Nonlinear Euler sums up to now. Another direction of investigation is the nonlinear case. In this case one considers sums like

$$
\sum_{n=1}^{\infty} \frac{H_{n, r_{1}} H_{n, r_{2}} \cdots H_{n, r_{p}}}{n^{a}}
$$

These are called nonlinear Euler sums. In this case just sporadic results are known; one can find some of them in the references [Borwein and Borwein 1995; Chu 1997; de Doelder 1991; Shen 1995; Sofo and Hassani 2012]. Moreover, V. Adamchik [1997] investigated the relation between such nonlinear Euler sums and several sums on the Stirling numbers of the fist kind. D. F. Connon [2008a; 2008b; 2008c; 2008d; 2008e; 2008f; 2008g; 2008h] has found a large number of connections between specific nonlinear Euler sums and the Riemann and Hurwitz zeta functions. To mention two beautiful results, we cite an expression for $\zeta(4)$ and $\zeta$ (5) [Connon 2008c, formulas (4.3.45f) and (4.3.57b)]:

$$
\zeta(4)=\frac{1}{6} \sum_{n=1}^{\infty} \frac{H_{n}^{2}+H_{n, 2}}{n^{2}}, \quad \zeta(5)=\frac{1}{24} \sum_{n=1}^{\infty} \frac{2 H_{n, 3}+3 H_{n} H_{n, 2}+H_{n}^{3}}{n^{2}} .
$$

To present some additional examples from the literature, we cite two sums from [Borwein and Borwein 1995]:

$$
\sum_{n=1}^{\infty} \frac{H_{n}^{2}}{n^{2}}=\frac{17}{4} \zeta(4), \quad \sum_{n=1}^{\infty} \frac{H_{n} H_{n+1}}{(n+1)^{2}}=3 \zeta(4)=\frac{\pi^{4}}{30}
$$


and from [Flajolet and Salvy 1998]:

$$
\begin{aligned}
\sum_{n=1}^{\infty} \frac{H_{n}^{3}}{n^{4}} & =\frac{231}{16} \zeta(7)-\frac{51}{4} \zeta(3) \zeta(4)+2 \zeta(2) \zeta(5), \\
\sum_{n=1}^{\infty} \frac{H_{n}^{4}}{(n+1)^{3}} & =\frac{185}{8} \zeta(7)-\frac{43}{2} \zeta(3) \zeta(4)+5 \zeta(2) \zeta(5) .
\end{aligned}
$$

That all of these sums can be expressed as multiple zeta values is not known. The only available result concerns quadratic sums:

Theorem 1 [Flajolet and Salvy 1998, p. 25, Theorem 4.2]. If $p_{1}+p_{2}+q$ is even, and $p_{1}>1, p_{2}>1, q>1$, the quadratic sums

$$
\sum_{n=1}^{\infty} \frac{H_{n, p_{1}} H_{n, p_{2}}}{n^{q}}
$$

are reducible to linear sums.

The theorem exactly gives the reduction, but the formulas are rather complicated to cite.

Finally, we cite a nice example of a nonlinear alternating sum from [Borwein and Borwein 1995]:

$\sum_{n=1}^{\infty} \frac{1}{n^{2}}\left(1-\frac{1}{2}+\cdots+\frac{(-1)^{n+1}}{n}\right)^{2}=-\frac{13}{8} \zeta(4)+\frac{5}{2} \zeta(2) \log ^{2} 2+\frac{1}{12} \log ^{4} 2+2 \operatorname{Li}_{4}\left(\frac{1}{2}\right)$.

Here

$$
\operatorname{Li}_{k}(z)=\sum_{n=1}^{\infty} \frac{z^{n}}{n^{k}}=\zeta(k ; z)
$$

is a special multiple zeta function, called the polylogarithm. (The special value $\mathrm{Li}_{4}\left(\frac{1}{2}\right)$, like $\zeta(3)$, does not seem to be evaluable in terms of more fundamental constants.)

No general reduction formula is known for nonlinear alternating sums, but computer-based calculations are available in several cases; see [Bailey et al. 1994].

An exhaustive and up-to-date bibliography on Euler sums and their generalizations can be found at http://www.usna.edu/Users/math/meh/biblio.html .

\section{New nonlinear Euler sum formulas}

Now we turn to our own results.

In this section we demonstrate how we can trace back some specific quadratic Euler sums to linear ones. To express these sums in a convenient form, we use the 
concept of E. A. Ulanskii, who defined the nonstrict multiple polylogarithm [2003] as follows:

$$
\operatorname{Le}_{\left(s_{1}, \ldots, s_{m}\right)}(z)=\sum_{1 \leq n_{m} \leq \cdots \leq n_{1}} \frac{z^{n_{1}}}{n_{1}^{S_{1}} n_{2}^{S_{2}} \cdots n_{m}^{s_{m}}} .
$$

As it can be seen, this definition differs from the multiple polylogarithm function in the nonstrictness of the relations. We shall always set the parameter $z$ to 1 , and we refer to the function

$$
\operatorname{Le}_{\left(s_{1}, \ldots, s_{m}\right)}(1)=: \operatorname{Le}\left(s_{1}, \ldots, s_{m}\right)
$$

as the nonstrict multiple zeta function. Ulanskii did not deal with the specific values of these sums but with the functional relations among them. For example, he proved the following theorem, which will be extremely useful for us.

Theorem 2. The nonstrict multiple polylogarithm function can be written as a sum of multiple polylogarithms as

$$
\operatorname{Le}_{\left(s_{1}, \ldots, s_{m}\right)}(z)=\sum_{\rho} \zeta(\rho ; z)
$$

where $\rho$ runs through all sets of the form $\left(s_{1} * \cdots * s_{m}\right)$, the symbol $*$ standing either for + or the comma; the total number of such sets is $2^{m-1}$. Moreover, $\zeta$ is defined under (2).

For further reference we specify this theorem with $m=2$ and $m=3$, with $z=1$ (in which form these relations has appeared in [Hoffman 1992]):

(7) $\operatorname{Le}\left(s_{1}, s_{2}, s_{3}\right)=\zeta\left(s_{1}, s_{2}, s_{3}\right)+\zeta\left(s_{1}+s_{2}+s_{3}\right)+\zeta\left(s_{1}, s_{2}+s_{3}\right)+\zeta\left(s_{1}+s_{2}, s_{3}\right)$.

Finally, we introduce the notations

$$
\begin{aligned}
H(a, b) & =\sum_{n=1}^{\infty} \frac{H_{n, b}}{n^{a}}, \\
H(a, b, c) & =\sum_{n=1}^{\infty} \frac{H_{n, b} H_{n, c}}{n^{a}}, \\
H(a, b, c, d) & =\sum_{n=1}^{\infty} \frac{H_{n, b} H_{n, c} H_{n, d}}{n^{a}} .
\end{aligned}
$$

We can call these sums ordinary (or first-order), quadratic (or second-order) and cubic Euler sums, respectively. 
3.1. Quadratic sums: the homogeneous case. We now show how we can trace back $H(a, b, b)$ to nonstrict multiple zeta function - and so, by Theorem 2, to multiple zeta values - and $H(a, b)$. Since there are extensive tables for multiple zeta values and many results for $H(a, b)$, we can calculate sums like

$$
H(a, b, b)=\sum_{n=1}^{\infty} \frac{H_{n, b}^{2}}{n^{a}}
$$

with a relatively small effort.

Theorem 3. For homogeneous quadratic Euler sums we have the reduction

$$
H(a, b, b)=2 \operatorname{Le}(a, b, b)-H(a, 2 b),
$$

or, if we write out the definitions,

$$
\sum_{n=1}^{\infty} \frac{H_{n, b}^{2}}{n^{a}}=2 \operatorname{Le}(a, b, b)-\sum_{n=1}^{\infty} \frac{H_{n, 2 b}}{n^{a}} .
$$

Proof. Let us write out the sums:

$$
H(a, b, b)=\sum_{n=1}^{\infty} \frac{H_{n, b}^{2}}{n^{a}}=\sum_{n=1}^{\infty} \frac{1}{n^{a}} \sum_{m=1}^{n} \frac{1}{m^{b}} \sum_{k=1}^{n} \frac{1}{k^{b}} .
$$

On the other hand,

$$
\operatorname{Le}(a, b, b)=\sum_{n=1}^{\infty} \frac{1}{n^{a}} \sum_{m=1}^{n} \frac{1}{m^{b}} \sum_{k=1}^{m} \frac{1}{k^{b}} .
$$

Geometrically, the sum $H(a, b, b)$ runs through a two dimensional square with integer coordinates. On the other hand, $\operatorname{Le}(a, b, b)$ runs through the lower triangle of this square, including the diagonal. By symmetry of the terms of the sums, the lattice points of this square are equal if we mirror them with respect to the main diagonal of the square. Therefore the sum $H(a, b, b)$ equals twice $\operatorname{Le}(a, b, b)$ minus the diagonal, which is counted twice. At the diagonal the inner sums equal

$$
\sum_{m=1}^{n} \frac{1}{m^{2 b}}
$$

Summing on the index $n$, we have our relation.

Employing the above theorem, in the next subsection we provide a concrete example. This example is chosen to be very typical. It uses almost all the usual tricks which lead to the zeta expression of an Euler sum. 
3.2. The homogeneous quadratic sums $H(2,2,2), H(2,1,1)$ and $H(3,1,1)$. According to the theorem of Flajolet and Salvy (in this paper Theorem 1), the sum

$$
\sum_{n=1}^{\infty} \frac{H_{n, 2}^{2}}{n^{2}}
$$

reduces to zeta values. Without using the evaluations of Flajolet and Salvy, we employ our above theorem. This implies the next representation.

Theorem 4.

$$
\sum_{n=1}^{\infty} \frac{H_{n, 2}^{2}}{n^{2}}=\frac{19}{24} \zeta(6)+\zeta^{2}(3) \text {. }
$$

Proof. The sum in the left-hand side equals

$$
H(2,2,2)=2 \operatorname{Le}(2,2,2)-H(2,4) .
$$

Our goal is to reduce the expression on the right to Riemann zeta values. By (7),

$$
\operatorname{Le}(2,2,2)=\zeta(6)+\zeta(4,2)+\zeta(2,4)+\zeta(2,2,2) .
$$

All the values $\zeta(4,2), \zeta(2,4)$ and $\zeta(2,2,2)$ can be found in [Li 2011]:

$$
\zeta(4,2)=\zeta^{2}(3)-\frac{4}{3} \zeta(6), \quad \zeta(2,4)=-\zeta^{2}(3)+\frac{25}{12} \zeta(6), \quad \zeta(2,2,2)=\frac{3}{16} \zeta(6)
$$

Altogether,

$$
\operatorname{Le}(2,2,2)=\frac{31}{16} \zeta(6)=\frac{31}{15120} \pi^{6}
$$

Now we deal with the sum $H(2,4)$. We could not find in the literature directly this sum, but in [Flajolet and Salvy 1998, p. 16, formula (b)] we can find that

$$
H(4,2)=\zeta^{2}(3)-\frac{1}{3} \zeta(6)
$$

We now apply the reflection formula [Boyadzhiev 2002; Flajolet and Salvy 1998]

$$
H(a, b)+H(b, a)=\zeta(a) \zeta(b)+\zeta(a+b)
$$

to obtain

$$
H(2,4)=\zeta(2) \zeta(4)+\zeta(6)-H(b, a)=\frac{37}{12} \zeta(6)-\zeta^{2}(3) .
$$

(Here we used the fact that $\zeta(2) \zeta(4)=\frac{7}{4} \zeta(6)$.) Hence

$$
H(2,2,2)=2 \cdot \frac{31}{16} \zeta(6)-\left(\frac{37}{12} \zeta(6)-\zeta^{2}(3)\right)=\frac{19}{24} \zeta(6)+\zeta^{2}(3) .
$$

This is what we wanted to prove. 
Connon [2007] gave an "elementary" evaluation for the sum $H(2,1,1)$, which was evaluated earlier by de Doelder [1991]. Their result is

$$
H(2,1,1)=\sum_{n=1}^{\infty} \frac{H_{n}^{2}}{n^{2}}=\frac{17}{4} \zeta(4) .
$$

Once we have Theorem 3, the evaluation of this sum reduces to looking for the appropriate values of the multiple zeta. These values can be found in [Borwein and Girgensohn 1996], hence our method gives a third (and the easiest) proof.

In the same paper Connon notes that he could not evaluate the sum $H(3,1,1)$. Our method and the multiple zeta values from the paper [Borwein and Girgensohn 1996] give immediately that

$$
H(3,1,1)=\sum_{n=1}^{\infty} \frac{H_{n}^{2}}{n^{3}}=\frac{7}{2} \zeta(5)-\zeta(2) \zeta(3) .
$$

However, Mathematica can evaluate this sum automatically.

Connon gave an integral representation for $H(q, 1,1)$ for integer $q>1$ :

$$
\sum_{n=1}^{\infty} \frac{H_{n}^{2}}{n^{q}}=\int_{0}^{1} \int_{0}^{1} \frac{\mathrm{Li}_{q-2}((1-t)(1-u)) \log t \log u}{(1-t)(1-u)} d u d t
$$

By the results above for $q=3$ and knowing that $\operatorname{Li}_{1}(x)=-\log (1-x)$, we get the closed form of the following integral:

$$
\int_{0}^{1} \int_{0}^{1} \frac{\log (1-(1-t)(1-u)) \log t \log u}{(1-t)(1-u)} d u d t=\zeta(2) \zeta(3)-\frac{7}{2} \zeta(5) .
$$

3.3. Quadratic sums: the inhomogeneous case. Another kind of approach helps us to evaluate inhomogeneous quadratic sums, i.e., sums of the form

$$
\sum_{n=1}^{\infty} \frac{H_{n, b} H_{n, c}}{n^{a}} .
$$

Namely, the next theorem is true.

Theorem 5. Inhomogeneous quadratic Euler sums can be expressed by the nonstrict multiple zeta function as

$$
H(a, b, c)=\operatorname{Le}(a, b, c)+\operatorname{Le}(a, c, b)-\operatorname{Le}(a, b+c) .
$$

If $c=b$ this formula reduces to the formula presented in Theorem 3. 


$$
\begin{aligned}
\text { Proof. } H(a, b, c) & =\sum_{n=1}^{\infty} \frac{H_{n, b} H_{n, c}}{n^{a}}=\sum_{n=1}^{\infty} \frac{1}{n^{a}}\left(\sum_{m=1}^{n} \frac{1}{m^{b}}\right)\left(\sum_{k=1}^{n} \frac{1}{k^{c}}\right) \\
& =\sum_{n=1}^{\infty} \frac{1}{n^{a}}\left(\sum_{m=1}^{n} \frac{1}{m^{b}}\right)\left(\sum_{k=1}^{m} \frac{1}{k^{c}}\right)+\sum_{n=1}^{\infty} \frac{1}{n^{a}}\left(\sum_{m=1}^{n} \frac{1}{m^{b}}\right)\left(\sum_{k=m+1}^{n} \frac{1}{k^{c}}\right) .
\end{aligned}
$$

The first sum is nothing but $\operatorname{Le}(a, b, c)$, while the second can be rearranged as

$$
\sum_{n=1}^{\infty} \frac{1}{n^{a}}\left(\sum_{m=1}^{n} \frac{1}{m^{b}}\right)\left(\sum_{k=m+1}^{n} \frac{1}{k^{c}}\right)=\sum_{n=1}^{\infty} \frac{1}{n^{a}}\left(\sum_{k=2}^{n} \frac{1}{k^{c}}\right)\left(\sum_{m=1}^{k-1} \frac{1}{m^{b}}\right) .
$$

Since the sum over $m$ is empty if $k=1$, we can start the sum over $k$ from 1 . Hence, at this point, we have that

$$
H(a, b, c)=\operatorname{Le}(a, b, c)+\sum_{n=1}^{\infty} \frac{1}{n^{a}}\left(\sum_{k=1}^{n} \frac{1}{k^{c}}\right)\left(\sum_{m=1}^{k-1} \frac{1}{m^{b}}\right) .
$$

The latter sum almost equals $\operatorname{Le}(a, c, b)$, but here the sum on $m$ runs up to $k-1$ instead of $k$. We can resolve this as follows:

$\sum_{n=1}^{\infty} \frac{1}{n^{a}}\left(\sum_{k=1}^{n} \frac{1}{k^{c}}\right)\left(\sum_{m=1}^{k-1} \frac{1}{m^{b}}\right)=\sum_{n=1}^{\infty} \frac{1}{n^{a}}\left(\sum_{k=1}^{n} \frac{1}{k^{c}}\right)\left(\sum_{m=1}^{k} \frac{1}{m^{b}}\right)-\sum_{n=1}^{\infty} \frac{1}{n^{a}}\left(\sum_{k=1}^{n} \frac{1}{k^{c+b}}\right)$.

The right-hand side equals $\operatorname{Le}(a, c, b)-\operatorname{Le}(a, c+b)$. Substituting this into the ultimate expression of $H(a, b, c)$, we are done.

3.4. Two nonhomogeneous quadratic sums: $H(2,1,2)$ and $H(2,2,3)$. By using the theorem of the last subsection, we evaluate the next inhomogeneous quadratic sums.

Theorem 6. We have

$$
\begin{aligned}
\sum_{n=1}^{\infty} \frac{H_{n} H_{n, 2}}{n^{2}} & =\zeta(2) \zeta(3)+\zeta(5), \\
\sum_{n=1}^{\infty} \frac{H_{n, 2} H_{n, 3}}{n^{2}} & =\frac{131}{16} \zeta(7)-\frac{5}{2} \zeta(2) \zeta(5)-\frac{3}{2} \zeta(3) \zeta(4) .
\end{aligned}
$$

Note that the theorem of Flajolet and Salvy does not apply to these sums. Proof. These proofs are again instructive. First,

$$
\sum_{n=1}^{\infty} \frac{H_{n} H_{n, 2}}{n^{2}}=H(2,1,2)=\operatorname{Le}(2,1,2)+\operatorname{Le}(2,2,1)-\operatorname{Le}(2,3) .
$$


Moreover, according to (7),

$$
\operatorname{Le}(2,1,2)=\zeta(5)+\zeta(3,2)+\zeta(2,3)+\zeta(2,1,2) .
$$

In [Borwein and Girgensohn 1996] we find that

$$
\zeta(2,1,2)=\frac{9}{2} \zeta(5)-2 \zeta(2) \zeta(3), \quad \zeta(3,2)=-\frac{11}{2} \zeta(5)+3 \zeta(2) \zeta(3) .
$$

We could not find the value of $\zeta(2,3)$ directly, but it can be easily deduced by a result of Boyadzhiev [2002]. Namely,

$$
\zeta(2,3)=\sum_{n=1}^{\infty} \frac{H_{n-1,3}}{n^{2}}=\sum_{n=1}^{\infty} \frac{H_{n, 3}}{n^{2}}-\zeta(5) .
$$

The zeta expression of the harmonic sum on the right — and even a more general form - is worked out by Boyadzhiev in the same paper:

$$
\sum_{n=1}^{\infty} \frac{H_{n, 3}}{n^{2}}=\frac{11}{2} \zeta(5)-2 \zeta(2) \zeta(3) .
$$

(In general, he found that

$$
\begin{aligned}
\sum_{n=1}^{\infty} \frac{H_{n, 3}}{n^{p}}=\zeta(3) \zeta(p) & +\left(1+\frac{p^{3}+5 p}{12}\right) \zeta(p+3)-\frac{1}{4} \sum_{k=1}^{p-1} k(k+1) \zeta(k+2) \zeta(p-k+1) \\
& -\frac{1}{4} p(p+1) H(p+2,1)-\frac{1}{2}[H(p+1,2)+\zeta(p+1) \zeta(2)],
\end{aligned}
$$

if $p$ is even.)

So

$$
\zeta(2,3)=\frac{9}{2} \zeta(5)-2 \zeta(2) \zeta(3)
$$

Altogether, we get that

$$
\operatorname{Le}(2,1,2)=\frac{9}{2} \zeta(5)-\zeta(2) \zeta(3)
$$

We also need the value of $\operatorname{Le}(2,2,1)$. Again, employing (7),

$$
\operatorname{Le}(2,2,1)=\zeta(5)+\zeta(4,1)+\zeta(2,3)+\zeta(2,2,1) .
$$

Using the tables in [Borwein and Girgensohn 1996], we find that

$$
\zeta(4,1)=2 \zeta(5)-\zeta(2) \zeta(3), \quad \zeta(2,2,1)=-\frac{11}{2} \zeta(5)+3 \zeta(2) \zeta(3) .
$$

Applying to (10) the value of $\zeta(2,3)$ calculated above, we get the simple zeta value of $\operatorname{Le}(2,2,1)$ :

$$
\operatorname{Le}(2,2,1)=2 \zeta(5)
$$


The last undetermined zeta value in (9) does not cause any problem:

$$
\operatorname{Le}(2,3)=\zeta(5)+\zeta(2,3)=\zeta(5)+\frac{9}{2} \zeta(5)-2 \zeta(2) \zeta(3)=\frac{11}{2} \zeta(5)-2 \zeta(2) \zeta(3) .
$$

Collecting the nonstrict zeta values in $(9)$ for $H(2,1,2)$, we find that they really sum to $\zeta(5)+\zeta(2) \zeta(3)$.

Now we turn to the second nonlinear Euler sum $H(2,2,3)$. It equals

$$
\operatorname{Le}(2,2,3)+\operatorname{Le}(2,3,2)-\operatorname{Le}(2,5) .
$$

We follow the same pattern as above:

$$
\operatorname{Le}(2,2,3)=\zeta(7)+\zeta(4,3)+\zeta(2,5)+\zeta(2,2,3) .
$$

The zeta expressions of $\zeta(4,3)$ and $\zeta(2,5)$ can be calculated from the results in [Bailey et al. 1994], under the notations $\sigma_{h}(3,4)$ and $\sigma_{h}(5,2)$, respectively. They equal

$$
\begin{aligned}
& \zeta(4,3)=17 \zeta(7)-10 \zeta(2) \zeta(5) \\
& \zeta(2,5)=10 \zeta(7)-2 \zeta(3) \zeta(4)-4 \zeta(2) \zeta(5)
\end{aligned}
$$

(In fact, the next expressions are deduced in [Bailey et al. 1994] and by the same authors in [Borwein et al. 1995]:

$$
\begin{aligned}
& \zeta(m, n)=\frac{1}{2}\left(\left(\begin{array}{c}
m+n \\
m
\end{array}\right)-1\right) \zeta(m+n)+\zeta(m) \zeta(n) \\
& \quad-\sum_{j=1}^{m+n}\left(\left(\begin{array}{c}
2 j-2 \\
m-1
\end{array}\right)+\left(\begin{array}{c}
2 j-2 \\
n-1
\end{array}\right)\right) \zeta(2 j-1) \zeta(m+n-2 j+1)
\end{aligned}
$$

if $m$ is odd and $n$ is even, while

$$
\begin{aligned}
\zeta(m, n)=-\frac{1}{2}\left(\left(\begin{array}{c}
m+n \\
m
\end{array}\right)+1\right) \zeta(m+n) \\
+\sum_{j=1}^{m+n}\left(\left(\begin{array}{c}
2 j-2 \\
m-1
\end{array}\right)+\left(\begin{array}{c}
2 j-2 \\
n-1
\end{array}\right)\right) \zeta(2 j-1) \zeta(m+n-2 j+1)
\end{aligned}
$$

if $m$ is even and $n$ is odd.)

The value of $\zeta(2,2,3)$ is listed in [Borwein and Girgensohn 1996]:

$$
\zeta(2,2,3)=-\frac{291}{16} \zeta(7)-\frac{3}{2} \zeta(3) \zeta(4)+12 \zeta(2) \zeta(5) .
$$

Hence

$$
\operatorname{Le}(2,2,3)=\frac{157}{16} \zeta(7)-2 \zeta(2) \zeta(5)-\frac{7}{2} \zeta(3) \zeta(4)
$$


The calculation of $\operatorname{Le}(2,3,2)$ needs a bit less work, because

$$
\operatorname{Le}(2,3,2)=\zeta(7)+\zeta(5,2)+\zeta(2,5)+\zeta(2,3,2),
$$

and we can apply the reflection formula [Wan 2012]

$$
\zeta(a, b)+\zeta(b, a)=\zeta(a) \zeta(b)-\zeta(a+b) .
$$

We have that

$$
\zeta(5,2)+\zeta(2,5)=\zeta(2) \zeta(5)-\zeta(7),
$$

and so

$$
\operatorname{Le}(2,3,2)=\zeta(2) \zeta(5)+\zeta(2,3,2)
$$

The value

$$
\zeta(2,3,2)=\frac{75}{8} \zeta(7)-\frac{11}{2} \zeta(2) \zeta(5)
$$

can be found in [Borwein and Girgensohn 1996]. Hence

$$
\operatorname{Le}(2,3,2)=\frac{75}{8} \zeta(7)-\frac{9}{2} \zeta(2) \zeta(5) .
$$

Only Le $(2,5)$ is missing in (11).

$$
\operatorname{Le}(2,5)=\zeta(7)+\zeta(2,5)=11 \zeta(7)-4 \zeta(2) \zeta(5)-2 \zeta(4) \zeta(3),
$$

as we can see from (13).

Substituting (15), (16), and (17) into (11), we have the second sum in Theorem 6.

3.5. Homogeneous cubic sums. The geometric approach we applied in Section 3.1 to homogeneous quadratic sums can be generalized to homogeneous cubic sums as well.

Theorem 7. The homogeneous cubic Euler sums can be reduced to multiple zeta values of depth 3 and 4, and to Euler sums of order one and two. Namely,

$$
H(a, b, b, b)=6 \zeta(a, b, b, b)+6 \zeta(a+b, b, b)+3 H(a, b, 2 b)-2 H(a, 3 b) .
$$

Proof. The sum

$$
H(a, b, b, b)=\sum_{n=1}^{\infty} \frac{1}{n^{a}} \sum_{m=1}^{n} \frac{1}{m^{b}} \sum_{k=1}^{n} \frac{1}{k^{b}} \sum_{l=1}^{n} \frac{1}{l^{b}}
$$

can be considered as a sum on the infinite cubic lattice with positive integer coordinates. We subtract from this the second-order sums on the principal planes $m=k, k=l$ and $m=l$. Since we have subtracted the main diagonal $m=k=l$ three times, we can add it two times. Then, by symmetry, we have six times the sum in the "lower" part of the cube, with (integer) coordinates $m=1,2, \ldots, n$, 
$k=1, \ldots, m-1$ and $l=1, \ldots, k-1$. Again, by symmetry, the second-order sums on the principal planes $m=k, k=l$ and $m=l$ are identical and equal $H(a, b, 2 b)$; and the main diagonal $m=k=l$ corresponds to the sum $H(a, 3 b)$. Hence, we have the relation

$$
\frac{H(a, b, b, b)-3 H(a, b, 2 b)+2 H(a, 3 b)}{6}=\sum_{n=1}^{\infty} \frac{1}{n^{a}} \sum_{m=1}^{n} \frac{1}{m^{b}} \sum_{k=1}^{m-1} \frac{1}{k^{b}} \sum_{l=1}^{k-1} \frac{1}{l^{b}} .
$$

The sum on the right-hand side can be easily rewritten as a multiple zeta expression, if we separate the terms $m=1,2, \ldots, n-1$ and $m=n$ :

$$
\sum_{n=1}^{\infty} \frac{1}{n^{a}} \sum_{m=1}^{n} \frac{1}{m^{b}} \sum_{k=1}^{m-1} \frac{1}{k^{b}} \sum_{l=1}^{k-1} \frac{1}{l^{b}}=\zeta(a, b, b, b)+\zeta(a+b, b, b) .
$$

Substituting this into the above relation and rearranging we have our theorem.

3.6. The inhomogeneous quadratic sum $H(4,1,2)$. We apply the theorem of the above section to prove the next identity.

Theorem 8. $\quad \sum_{n=1}^{\infty} \frac{H_{n} H_{n, 2}}{n^{4}}=\frac{3}{4} \zeta(3) \zeta(4)+2 \zeta(2) \zeta(5)-\frac{51}{16} \zeta(7)$.

Note that here the reduction theorem of Flajolet and Salvy does not apply.

Proof. We specialize Theorem 7 to $a=4$ and $b=1$. Then

$$
H(4,1,1,1)=6 \zeta(4,1,1,1)+6 \zeta(5,1,1)+3 H(4,1,2)-2 H(4,3) .
$$

The sum on the left-hand side equals

$$
H(4,1,1,1)=\sum_{n=1}^{\infty} \frac{H_{n}^{3}}{n^{4}}=\frac{231}{16} \zeta(7)-\frac{51}{4} \zeta(3) \zeta(4)+2 \zeta(2) \zeta(5),
$$

as one can find in [Flajolet and Salvy 1998, p. 16]. Moreover, an important simplification can be done on the right-hand side, since

$$
\zeta(4,1,1,1)=\zeta(5,1,1) .
$$

This is an observation of J. Borwein, D. Bradley and D. Broadhurst, see the paragraph after formula (30) in [1997]. The general version that they proved is the following:

$$
\zeta\left(m+2,\{1\}_{n}\right)=\zeta\left(n+2,\{1\}_{m}\right),
$$

where $\{1\}_{n}$ means that we repeat the argument $n$ times. Identity (20) comes if we substitute $m=2$ and $n=3$. Other examples are

$$
\zeta\left(2,\{1\}_{n}\right)=\zeta(n+2), \quad \zeta\left(3,\{1\}_{n}\right)=\zeta(n+2,1),
$$


and so on.

The value of $\zeta(5,1,1)$ can be found in [Borwein and Girgensohn 1996]:

$$
\zeta(5,1,1)=-\frac{5}{4} \zeta(3) \zeta(4)+5 \zeta(7)-2 \zeta(5) \zeta(2) .
$$

Thus, with respect to (18) and (19) we have the temporary result

$$
\begin{aligned}
& \frac{231}{16} \zeta(7)-\frac{51}{4} \zeta(3) \zeta(4)+2 \zeta(2) \zeta(5) \\
&=-15 \zeta(3) \zeta(4)+60 \zeta(7)-24 \zeta(5) \zeta(2)+3 H(4,1,2)-2 H(4,3),
\end{aligned}
$$

which can be rearranged:

$$
3 H(4,1,2)=-\frac{729}{16} \zeta(7)+\frac{9}{4} \zeta(3) \zeta(4)+26 \zeta(2) \zeta(5)+2 H(4,3) .
$$

The sum $H(4,3)$ can be deduced from the formula of Bailey, Borwein, and Girgensohn (14):

$$
H(4,3)=\sum_{n=1}^{\infty} \frac{H_{n, 3}}{n^{4}}=\sum_{n=0}^{\infty} \frac{H_{n+1,3}}{(n+1)^{4}}=\sum_{n=0}^{\infty} \frac{H_{n, 3}+1 /(n+1)^{3}}{(n+1)^{4}}=\zeta(4,3)+\zeta(7) .
$$

By using (12),

$$
H(4,3)=18 \zeta(7)-10 \zeta(2) \zeta(5) .
$$

Substituting this into (21), we are done.

\section{Generating functions of nonlinear Euler sums}

Up to this point, we were interested in closed form expression for quadratic and cubic Euler sums. In several cases, using polylogarithms and several tricks, we can involve a free parameter $z$ in these sums and express them with known special functions. To be more concrete, we can find the generating functions for $H_{n}^{2}$ and $H_{n}^{3}$ as well. We shall deduce the formulas in the next theorem.

Theorem 9. For any $|z|<1$ the ordinary generating functions of $H_{n}^{2}$ and $H_{n}^{3}$ are

$$
\begin{aligned}
& \sum_{n=1}^{\infty} H_{n}^{2} z^{n}=\frac{1}{1-z}\left(\operatorname{Li}_{2}(z)+\log ^{2}(1-z)\right) \\
& \begin{aligned}
\sum_{n=1}^{\infty} H_{n}^{3} z^{n}= & \frac{1}{1-z}\left(-\frac{\pi^{2}}{2} \log (1-x)-\log ^{3}(1-z)+\frac{3}{2} \log ^{2}(1-z) \log z\right. \\
& \left.+3 \operatorname{Li}_{3}(1-z)+\operatorname{Li}_{3}(z)-3 \zeta(3)\right)
\end{aligned}
\end{aligned}
$$

The first relation is easy to prove and is not new; one can find it, for example, in [Mező 2013]. For the sake of completeness, we give its proof. To our knowledge, the second formula is new. 
Proof. Note that $H_{n-1}^{2}=\left(H_{n}-\frac{1}{n}\right)^{2}=H_{n}^{2}+\frac{1}{n^{2}}-2 \frac{H_{n}}{n}$, whence

$$
\sum_{n=1}^{\infty} H_{n-1}^{2} z^{n}=\sum_{n=1}^{\infty} H_{n}^{2} z^{n}+\sum_{n=1}^{\infty} \frac{z^{n}}{n^{2}}-2 \sum_{n=1}^{\infty} \frac{H_{n}}{n} z^{n} .
$$

The second sum is $\operatorname{Li}_{2}(z)$ (see definition (5)), while the last sum equals

$$
\sum_{n=1}^{\infty} \frac{H_{n}}{n} z^{n}=\mathrm{Li}_{2}(z)+\frac{1}{2} \log ^{2}(1-z),
$$

by [Borwein and Borwein 1995]. If we temporarily introduce the function

$$
f(z)=\sum_{n=1}^{\infty} H_{n}^{2} z^{n}
$$

then (24) and (25) imply that

$$
z f(z)=f(z)+\mathrm{Li}_{2}(z)-2\left(\operatorname{Li}_{2}(z)+\frac{1}{2} \log ^{2}(1-z)\right) ;
$$

hence

$$
f(z)=\sum_{n=1}^{\infty} H_{n}^{2} z^{n}=\frac{\operatorname{Li}_{2}(z)+\log ^{2}(1-z)}{1-z} .
$$

Let us prove the second formula. Our initial point is almost the same as above:

$$
H_{n}^{3}=\left(H_{n-1}+\frac{1}{n}\right)^{3}=H_{n-1}^{3}+3 H_{n-1}^{2} \frac{1}{n}+3 H_{n-1} \frac{1}{n^{2}}+\frac{1}{n^{3}} .
$$

This time we set

$$
f(z)=\sum_{n=1}^{\infty} H_{n}^{3} z^{n}
$$

Then

$$
f(z)=z f(z)+3 \sum_{n=0}^{\infty} \frac{H_{n}^{2}}{n+1} z^{n+1}+3 \sum_{n=0}^{\infty} \frac{H_{n}}{(n+1)^{2}} z^{n+1}+\mathrm{Li}_{3}(z) .
$$

To calculate the first sum, we utilize the first formula of the theorem:

$$
\begin{aligned}
\sum_{n=0}^{\infty} \frac{H_{n}^{2}}{n+1} z^{n+1} & =\int_{0}^{z}\left(\frac{\operatorname{Li}_{2}(x)+\log ^{2}(1-x)}{1-x}\right) d x \\
& =-\frac{\pi^{2}}{3} \log (1-z)-\frac{1}{3} \log ^{3}(1-z)+\log ^{2}(1-z) \log z \\
& \quad+\log (1-z) \operatorname{Li}_{2}(z)+2 \operatorname{Li}_{3}(1-z)-2 \zeta(3) .
\end{aligned}
$$

This can be seen directly by differentiation. The integration constant $2 \zeta(3)$ comes if we substitute $z=0$. 
Now we deal with the second sum on the right-hand side of (26).

(28)

$$
\begin{aligned}
& \sum_{n=1}^{\infty} \frac{H_{n}}{(n+1)^{2}} z^{n+1} \\
& \quad=\int_{0}^{z} \sum_{n=1}^{\infty} \frac{H_{n}}{n+1} x^{n} d x=\int_{0}^{z} \frac{\log ^{2}(1-x)}{2 x} d x \\
& \quad=\frac{1}{2} \log ^{2}(1-z) \log z+\log (1-z) \operatorname{Li}_{2}(1-z)-\operatorname{Li}_{3}(1-z)+\zeta(3) .
\end{aligned}
$$

Taking the derivative of the right-hand side, this can be justified. The integration constant comes if we substitute $z=0$.

Substituting (27) and (28) into (26) and utilizing [Lewin 1991, formula (1.5)]

$$
\mathrm{Li}_{2}(z)+\mathrm{Li}_{2}(1-z)=\zeta(2)-\log z \log (1-z)
$$

to simplify, we have proven Theorem 9.

It is interesting that $\mathrm{S}$. Ramanujan dealt with a similar function as in (28), but in the denominator there is $(n+1)^{3}$ in place of $(n+1)^{2}$ in his function:

$$
h(z)=\sum_{n=1}^{\infty} \frac{H_{n}}{(n+1)^{3}} z^{n+1} .
$$

He could not provide a closed form for this function but he showed that it can be analytically continued to the whole complex plane in $z$ and proved some functional equations for $h$. Details can be found in [Berndt 1985, p. 253]. Such generating functions also appear in a beautiful paper of Guillera and Sondow [2008].

4.1. Some series as consequences of Theorem 9. We note an interesting alternating nonlinear sum as a corollary of formula (27) in the proof of Theorem 9:

$$
\sum_{n=1}^{\infty}(-1)^{n+1} \frac{H_{n}^{2}}{n+1}=\frac{\pi^{2}}{12} \log 2-\frac{1}{3} \log ^{3} 2-\frac{1}{4} \zeta(3) .
$$

The proof can be done by substituting $z=-1$ into (27) and handling the occurring imaginary values. One of them is $\log (-1)$, the other one is $\mathrm{Li}_{3}(2)$. By a formula of Lewin's book [1981, (6.7), p. 154],

$$
\operatorname{Li}_{3}(2)=\mathrm{Li}_{3}\left(\frac{1}{2}\right)+\frac{\pi^{2}}{3} \log 2-\frac{1}{6} \log ^{3} 2-\frac{1}{2} i \pi \log ^{2} 2 .
$$

Since $\log (-1)=i \pi$ in the principal branch, the imaginary parts cancel - as they must - and then we can finish the proof using the special values

$$
\operatorname{Li}_{2}(-1)=-\frac{\pi^{2}}{12}, \quad \operatorname{Li}_{3}\left(\frac{1}{2}\right)=\frac{1}{24}\left(4 \log ^{3}(2)+21 \zeta(3)-2 \pi^{2} \log 2\right) .
$$


Another consequence of the calculations in (28) is the classic result of Euler, which is nothing but (1):

$$
\sum_{n=1}^{\infty} \frac{H_{n}}{(n+1)^{2}}=\zeta(3)
$$

To prove this we let $z$ tend to 1 from the left. (Taking the limit is not straightforward, we have to check the Taylor series around 1 to see that we have the right to do this. Finally we see that all the terms cancel, and just the constant term $\zeta(3)$ remains.)

Nice sums of infinite series involving the square and third power of the digamma function are consequences of Theorem 9. This function is the logarithmic derivative of the Euler $\Gamma$ function and can be defined by the sum [Gradshteyn and Ryzhik 2007]

$$
\psi(x)=-\gamma+\sum_{n=0}^{\infty}\left(\frac{1}{n+1}-\frac{1}{n+x}\right) \quad(x \in \mathbb{R} \backslash\{0,-1,-2, \ldots\}) .
$$
Here $\gamma=-\lim _{n \rightarrow \infty}\left(\log n-\sum_{k=1}^{n} \frac{1}{k}\right) \approx 0.577215664901533$ is the Euler-Mascheroni
constant.

The derivatives of the digamma function $\psi^{\prime}, \psi^{\prime \prime}, \ldots$ are called trigamma, tetragamma functions, etc. In general, these derivatives are called polygamma functions and denoted by $\psi_{n}\left(\psi_{0}=\psi, \psi_{1}=\psi^{\prime}, \ldots\right)$. Since

$$
\psi_{k}(n)=(-1)^{k+1} k !\left(\zeta(k+1)-H_{n-1, k+1}\right),
$$

it is straightforward to see that the polygamma functions have the generating functions

$$
\sum_{n=1}^{\infty} \psi_{k}(n) z^{n}=\frac{z}{1-z}(-1)^{k} k !\left(\operatorname{Li}_{k+1}(z)-\zeta(k+1)\right) \quad(|z|<1, k=1,2, \ldots) .
$$

If $k=0$, we have that

$$
\sum_{n=1}^{\infty} \psi(n) z^{n}=\frac{z}{z-1}(\gamma+\log (1-z)) .
$$

From the general representation (29) it follows that at a positive integer $n$ the digamma function equals

$$
\psi(n)=H_{n-1}-\gamma .
$$

We have infinite series for the second and third power of the digamma function:

$$
\sum_{n=1}^{\infty} \frac{\psi^{2}(n+1)}{2^{n}}=\gamma^{2}-4 \gamma \log 2+\log ^{2} 2+\zeta(2),
$$


$\sum_{n=1}^{\infty} \frac{\psi^{3}(n+1)}{2^{n}}=\frac{\pi^{2}}{3} \log 2+\frac{1}{3} \log ^{3} 2+\zeta(3)-\frac{\pi^{2}}{2} \gamma-3 \gamma \log ^{2} 2+6 \gamma^{2} \log 2-\gamma^{3}$.

It is interesting that the second formula includes all the most frequently appearing constants: $\pi, \gamma, e, \zeta(3)$, and $\log 2$.

We shall prove just the second identity, because the first one is similar but simpler. Using Theorem 9, we can see that

$$
\sum_{n=1}^{\infty} \frac{H_{n}^{3}}{2^{n}}=\frac{\pi^{2}}{3} \log 2+\frac{1}{3} \log ^{3} 2+\zeta(3)
$$

and

$$
\sum_{n=1}^{\infty} \frac{H_{n}^{2}}{2^{n}}=\zeta(2)+\log ^{2} 2=\frac{\pi^{2}}{6}+\log ^{2} 2 .
$$

From the generating function

$$
\sum_{n=1}^{\infty} H_{n} z^{n}=-\frac{\log (1-z)}{1-z}
$$

it is obvious that

$$
\sum_{n=1}^{\infty} \frac{H_{n}}{2^{n}}=\log 4
$$

Since

$$
\psi^{3}(n+1)=H_{n}^{3}-3 \gamma H_{n}^{2}+3 \gamma^{2} H_{n}-\gamma^{3},
$$

the result follows after dividing by $2^{n}$ and summing over $n$.

\section{The Landen functional equations of the dilogarithm and trilogarithm functions}

As an application of generating functions of the above nonlinear Euler sums we give proofs for the functional equations of the dilogarithm and trilogarithm functions. The proof relies on finite identities and on a result of Euler with respect to binomial transforms.

More concretely, we shall reprove the functional equation of the dilogarithm function:

$$
\operatorname{Li}_{2}\left(\frac{x}{1+x}\right)=-\frac{1}{2} \log ^{2}(1+x)-\operatorname{Li}_{2}(-x) .
$$

This is called Landen's equation [Lewin 1981, (1.12), p. 5]. 
We also show a new proof of the Landen functional equation for the trilogarithm:

$$
\begin{aligned}
\operatorname{Li}_{3}\left(\frac{x}{1+x}\right)=\zeta(3)+\zeta(2) \log (1 & +x)-\frac{1}{2} \log ^{2}(1+x) \log (-x) \\
& +\frac{1}{6} \log ^{3}(1+x)-\mathrm{Li}_{3}(-x)+\mathrm{Li}_{3}(1+x) .
\end{aligned}
$$

This is proved in [Lewin 1981, p. 155].

We remark that these equations are also presented in [Lewin 1991] on p. 2, but there is a typo there: in equation (1.13) in place of the coefficient $\frac{1}{6}$ there is $\frac{1}{2}$, which is incorrect.

The known proofs are analytic. We present proofs which are based on finite combinatorial identities. Moreover, we show a reason why there probably does not exist a functional equation of Landen type for higher-order polylogarithms.

Closed-form expressions for $\operatorname{Li}_{2}\left(\frac{1}{2}\right)$ and $\operatorname{Li}_{3}\left(\frac{1}{2}\right)$ are also known [Lewin 1991, pages 1 and 2]:

$$
\operatorname{Li}_{2}\left(\frac{1}{2}\right)=\frac{\pi^{2}}{12}-\frac{1}{2} \log ^{2} 2, \quad \operatorname{Li}_{3}\left(\frac{1}{2}\right)=\frac{7}{8} \zeta(3)-\frac{\pi^{2}}{12} \log 2-\frac{1}{6} \log ^{3} 2 .
$$

But there is no such formula for $\operatorname{Li}_{4}\left(\frac{1}{2}\right)$; see the remark after equation (7.92) in [Lewin 1981, p. 211].

We try to get closer to the constant $\operatorname{Li}_{4}\left(\frac{1}{2}\right)$ and we show that

$$
\begin{aligned}
& \mathrm{Li}_{4}\left(\frac{1}{2}\right) \\
& =\frac{\pi^{4}}{180}+\frac{\pi^{2}}{48} \log ^{2} 2-\frac{1}{24} \log ^{4} 2-\frac{7}{16} \log (2) \zeta(3)+\frac{1}{2} \sum_{n=1}^{\infty}(-1)^{n+1} \frac{H_{n} H_{n, 2}}{n} .
\end{aligned}
$$

The last sum on the right does not seem to be reducible to known constants. When we tried to reduce it, we found that in its expression $\operatorname{Li}_{4}\left(\frac{1}{2}\right)$ appears, so we would get a $0=0$-type identity upon substituting this into (33).

The new proofs of the Landen identities are based on the representations of the generalized harmonic numbers:

$$
\begin{aligned}
& H_{n, 2}=\sum_{k=1}^{n}\left(\begin{array}{l}
n \\
k
\end{array}\right)(-1)^{k+1} \frac{H_{k}}{k}, \\
& H_{n, 3}=\frac{1}{2} \sum_{k=1}^{n}\left(\begin{array}{l}
n \\
k
\end{array}\right) \frac{(-1)^{k+1}}{k}\left(H_{k}^{2}+H_{k, 2}\right), \\
& H_{n, 4}=\frac{1}{6} \sum_{k=1}^{n}\left(\begin{array}{l}
n \\
k
\end{array}\right) \frac{(-1)^{k+1}}{k}\left(H_{k}^{3}+3 H_{n} H_{n, 2}+2 H_{n, 3}\right),
\end{aligned}
$$

for all $n \geq 1$. (It is interesting that in the last sum, the term $H_{k}^{3}+3 H_{n} H_{n, 2}+2 H_{n, 3}$ appears in [Adamchik 1997; Connon 2008a]. To see how to derive identities like this, we refer to [Connon 2008c].) 
To prove (31) and (32) we need an identity, due to Euler, giving the generating function of a sequence's binomial transform. Recall that for an arbitrary real sequence $a_{n}$, the binomial transform of $a_{n}$ is the sequence $b_{n}$ defined by

$$
b_{n}=\sum_{k=0}^{n}\left(\begin{array}{l}
n \\
k
\end{array}\right) a_{k}, \quad \text { or, equivalently, } \quad a_{n}=\sum_{k=0}^{n}\left(\begin{array}{l}
n \\
k
\end{array}\right)(-1)^{n-k} b_{k} .
$$
If $a_{n}$ has the generating function $a(x)$, that is, $\sum_{n=0}^{\infty} a_{n} x^{n}=a(x)$, then $b_{n}$ has the
generating function

$$
\sum_{n=0}^{\infty} b_{n} x^{n}=\frac{1}{1-x} a\left(\frac{x}{1-x}\right) .
$$

For more information on binomial transforms and Euler's result, see [Dumont 1981; Mező and Dil 2009; Seidel 1877].

5.1. The Landen equation for the dilogarithm. It is straightforward to see that

$$
\sum_{n=1}^{\infty} H_{n, k} x^{n}=\frac{\operatorname{Li}_{k}(x)}{1-x}
$$

and from (34) we also know that $H_{n, 2}$ is the inverse binomial transform of $-H_{n} / n$. Hence

$$
\frac{\mathrm{Li}_{2}(x)}{1-x}=\frac{1}{1-x} a\left(\frac{x}{1-x}\right),
$$

where $a(x)$ is the generating function of $H_{n} / n$. The denominator $1+x$ cancels, and we apply the substitution $x \rightarrow x /(1+x)$ to get

$$
\operatorname{Li}_{2}\left(\frac{x}{1+x}\right)=a(x)
$$

Finally, to prove (31) we realize that

$$
\begin{aligned}
a(x)=-\sum_{n=1}^{\infty} \frac{(-1)^{n}}{n} H_{n} x^{n} & =-\sum_{n=1}^{\infty} \frac{(-1)^{n}}{n}\left(H_{n-1}+\frac{1}{n}\right) x^{n} \\
& =-\sum_{n=1}^{\infty} \frac{(-1)^{n}}{n} H_{n-1} x^{n}-\sum_{n=1}^{\infty} \frac{(-1)^{n}}{n^{2}} x^{n}
\end{aligned}
$$

The last two sums equal respectively $\frac{1}{2} \log ^{2}(1+x)$ and $\operatorname{Li}_{2}(-x)$ (in the latter case by definition). These prove (31).

5.2. The Landen equation for the trilogarithm. Identity (35) shows that

$$
\operatorname{Li}_{3}\left(\frac{x}{1+x}\right)=-\frac{1}{2} \sum_{n=1}^{\infty} \frac{(-1)^{n}}{n} H_{n}^{2} x^{n}-\frac{1}{2} \sum_{n=1}^{\infty} \frac{(-1)^{n}}{n} H_{n, 2} x^{n} .
$$


Let us deal with the first sum. We prove that

$$
\begin{aligned}
\sum_{n=1}^{\infty} \frac{H_{n}^{2}}{n} x^{n}=\mathrm{Li}_{3}(x) & +2 \operatorname{Li}_{2}(1-x) \log (1-x)+\mathrm{Li}_{2}(x) \log (1-x) \\
& -\frac{1}{3} \log ^{3}(1-x)+2 \log x \log ^{2}(1-x)-\frac{1}{3} \pi^{2} \log (1-x)
\end{aligned}
$$

for all $|x|<1$.

Applying (22), we have that

$$
\sum_{n=1}^{\infty} \frac{H_{n}^{2}}{n} x^{n}=\int_{0}^{x} \frac{\operatorname{Li}_{2}(y)}{y(1-y)} d y+\int_{0}^{x} \frac{\log ^{2}(y)}{y(1-y)} d y .
$$

These integrands have primitive functions:

$$
\begin{array}{r}
\int_{0}^{x} \frac{\operatorname{Li}_{2}(y)}{y(1-y)} d y=2 \mathrm{Li}_{3}(1-x)+\operatorname{Li}_{3}(x)+\operatorname{Li}_{2}(x) \log (1-x) \\
+\log x \log ^{2}(1-x)-\frac{1}{3} \pi^{2} \log (1-x)-2 \zeta(3),
\end{array}
$$

and

$$
\begin{array}{r}
\int_{0}^{x} \frac{\log ^{2}(y)}{y(1-y)} d y=-2 \operatorname{Li}_{3}(1-x)+2 \operatorname{Li}_{2}(1-x) \log (1-x) \\
-\frac{1}{3} \log ^{3}(1-x)+\log x \log ^{2}(1-x)+2 \zeta(3),
\end{array}
$$

as can be seen by differentiation. (The integration constants come if we substitute $x=0$.) These two integrals together give (38).

Similarly,

$$
\sum_{n=1}^{\infty} \frac{H_{n, 2}}{n} x^{n}=\int_{0}^{x} \frac{\mathrm{Li}_{2}(y)}{y(1-y)} d y .
$$

This integral is the same as (39).

Collecting the results under (38) and (41) (considering (39)) and putting them into (37), we get the Landen equation for the trilogarithm, after a simplification.

5.3. The Landen equation for the tetralogarithm and higher-order polylogs. Let us go to the tetralogarithm $\mathrm{Li}_{4}(x)$. Identity (36) immediately gives

$$
\mathrm{Li}_{4}\left(\frac{x}{1+x}\right)=\frac{1}{6} \sum_{n=1}^{\infty} \frac{(-1)^{n+1}}{n}\left(H_{n}^{3}+3 H_{n} H_{n, 2}+2 H_{n, 3}\right) x^{n} .
$$

This shows why finding a functional equation of Landen type for $\operatorname{Li}_{4}(x)$ is not hopeful: the product $H_{n} H_{n, 2}$ does not seem to have a generating function expressible by standard functions for all $|x|<1$. This is probably true for higher-order polylogarithms as well, because those harmonic number expressions probably contain $H_{n}^{4}$ and other powers and products of generalized harmonic numbers. 
We note that Theorem 1 of [Ulanskii 2003] does provide a general Landen functional equation for these polylogarithms. However, that equation uses multiple zeta functions, which probably cannot be reduced to polylogarithms and ordinary logarithms.

\section{Collected sums}

We close the paper collecting the calculated sums.

$$
\begin{aligned}
& \sum_{n=1}^{\infty} \frac{H_{n}^{2}}{n^{2}}=\frac{17}{4} \zeta(4), \quad \sum_{n=1}^{\infty} \frac{H_{n}^{2}}{n^{3}}=\frac{7}{2} \zeta(5)-\zeta(2) \zeta(3), \\
& \sum_{n=1}^{\infty} \frac{H_{n, 2}^{2}}{n^{2}}=\frac{19}{24} \zeta(6)+\zeta^{2}(3), \quad \sum_{n=1}^{\infty} \frac{H_{n} H_{n, 2}}{n^{2}}=\zeta(2) \zeta(3)+\zeta(5), \\
& \sum_{n=1}^{\infty} \frac{H_{n, 2} H_{n, 3}}{n^{2}}=\frac{131}{16} \zeta(7)-\frac{5}{2} \zeta(2) \zeta(5)-\frac{3}{2} \zeta(3) \zeta(4), \\
& \sum_{n=1}^{\infty} \frac{H_{n} H_{n, 2}}{n^{4}}=\frac{3}{4} \zeta(3) \zeta(4)+2 \zeta(2) \zeta(5)-\frac{51}{16} \zeta(7), \\
& \sum_{n=1}^{\infty}(-1)^{n+1} \frac{H_{n}^{2}}{n+1}=\frac{\pi^{2}}{12} \log 2-\frac{1}{3} \log ^{3} 2-\frac{1}{4} \zeta(3), \\
& \sum_{n=1}^{\infty} \frac{H_{n}^{3}}{2^{n}}=\frac{\pi^{2}}{3} \log 2+\frac{1}{3} \log ^{3}(2)+\zeta(3), \quad \sum_{n=1}^{\infty} \frac{H_{n}^{2}}{2^{n}}=\zeta(2)+\log ^{2} 2 .
\end{aligned}
$$

We also present some other sums without proof. The methods of Sections 4 and 5 can help get these as well.

$$
\begin{aligned}
& \sum_{n=1}^{\infty} \frac{(-1)^{n+1}}{n} H_{n}^{3}=\frac{1}{144}\left(\pi^{4}+18 \pi^{2} \log ^{2} 2-36 \log ^{4} 2+162 \log (2) \zeta(3)\right) \\
& \sum_{n=1}^{\infty} \frac{(-1)^{n+1}}{n} H_{n, 3}=\frac{19}{1440} \pi^{4}-\frac{3}{4} \log (2) \zeta(3) \\
& \sum_{n=1}^{\infty} \frac{(-1)^{n}}{n} H_{n-1} H_{n-1,2}=\frac{7}{8} \log (2) \zeta(3)-\frac{1}{4} \log ^{2}(2) \zeta(2)-\frac{1}{8} \zeta^{2}(2) \\
& \sum_{n=1}^{\infty} \frac{(-1)^{n}}{n} H_{n} H_{n, 2}=\zeta(4)-\frac{1}{12} \log ^{4} 2-2 \operatorname{Li}_{4}\left(\frac{1}{2}\right)+\frac{\pi^{2}}{24} \log ^{2} 2-\frac{7}{8} \log (2) \zeta(3)
\end{aligned}
$$


To conclude, we record the amusing identity of sums

$$
\sum_{n=1}^{\infty} H_{n, 2} \frac{(-1)^{n}}{n !}=\frac{\pi^{2}}{6 e}-\sum_{n=0}^{\infty} \frac{1}{(n+1)^{2}} \frac{! n}{n !} .
$$

Here $! n$ is the subfactorial of $n$ (the number of permutations on $n$ elements that don't fix any of them) and $e=\exp (1)$. The reader can look for a proof as a challenge.

\section{Acknowledgement}

The author is grateful to the referee, who recommended some additional literature. Thanks to this, the integral (8) now has a closed form evaluation.

\section{References}

[Adamchik 1997] V. Adamchik, “On Stirling numbers and Euler sums", J. Comput. Appl. Math. 79:1 (1997), 119-130. MR 97m:11025 Zbl 0877.39001

[Andrews et al. 1999] G. E. Andrews, R. Askey, and R. Roy, Special functions, Encyclopedia of Mathematics and its Applications 71, Cambridge University Press, 1999. MR 2000g:33001 Zbl 0920.33001

[Apostol and Vu 1984] T. M. Apostol and T. H. Vu, "Dirichlet series related to the Riemann zeta function”, J. Number Theory 19:1 (1984), 85-102. MR 85j:11106 Zbl 0539.10032

[Bailey et al. 1994] D. H. Bailey, J. M. Borwein, and R. Girgensohn, "Experimental evaluation of Euler sums", Experiment. Math. 3:1 (1994), 17-30. MR 96e:11168 Zbl 0810.11076

[Berndt 1985] B. C. Berndt, Ramanujan's notebooks, vol.1, Springer, New York, 1985. MR 86c:01062 Zbl 0555.10001

[Bigotte et al. 2002] M. Bigotte, G. Jacob, N. E. Oussous, and M. Petitot, "Lyndon words and shuffle algebras for generating the coloured multiple zeta values relations tables", Theoret. Comput. Sci. 273:1-2 (2002), 271-282. MR 2003j:11097 Zbl 1014.68126

[Borwein and Borwein 1995] D. Borwein and J. M. Borwein, "On an intriguing integral and

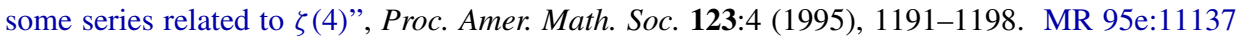
Zbl 0840.11036

[Borwein and Bradley 2006] J. M. Borwein and D. M. Bradley, "Thirty-two Goldbach variations", Int. J. Number Theory 2:1 (2006), 65-103. MR 2007e:11109 Zbl 1094.11031

[Borwein and Girgensohn 1996] J. M. Borwein and R. Girgensohn, "Evaluation of triple Euler sums", Electron. J. Combin. 3:1 (1996), R23, 1-27. MR 97d:11137 Zbl 0884.40005

[Borwein et al. 1995] D. Borwein, J. M. Borwein, and R. Girgensohn, "Explicit evaluation of Euler sums", Proc. Edinburgh Math. Soc. (2) 38:2 (1995), 277-294. MR 96f:11106 Zbl 0819.40003

[Borwein et al. 1997] J. M. Borwein, D. M. Bradley, and D. J. Broadhurst, "Evaluations of $k$-fold Euler/Zagier sums: A compendium of results for arbitrary k", Electron. J. Combin. 4:2 (1997), R5, 1-21. MR 98b:11091 Zbl 0884.40004

[Bowman and Bradley 2001] D. Bowman and D. M. Bradley, "Multiple polylogarithms: A brief survey", pp. 71-92 in q-series with applications to combinatorics, number theory, and physics (Urbana, IL, 2000), edited by B. C. Berndt and K. Ono, Contemp. Math. 291, Amer. Math. Soc., Providence, RI, 2001. Also available on arXiv: math/0310062. MR 2003c:33021 Zbl 0998.33013 
[Boyadzhiev 2002] K. N. Boyadzhiev, "Consecutive evaluation of Euler sums", Int. J. Math. Math. Sci. 29:9 (2002), 555-561. MR 2003d:11124 Zbl 0994.40003

[Boyadzhiev et al. 2008] K. N. Boyadzhiev, H. G. Gadiyar, and R. Padma, "The values of an Euler sum at the negative integers and a relation to a certain convolution of Bernoulli numbers", Bull. Korean Math. Soc. 45:2 (2008), 277-283. MR 2009e:11174 Zbl 1148.11046

[Boyadzhiev et al. 2009] K. N. Boyadzhiev, H. Gopalkrishna Gadiyar, and R. Padma, "Alternating Euler sums at the negative integers", Hardy-Ramanujan J. 32 (2009), 24-37. MR 2010f:11144 Zbl 1194.11090

[Briggs et al. 1955] W. E. Briggs, S. Chowla, A. J. Kempner, and W. E. Mientka, "On some infinite series", Scripta Math. 21 (1955), 28-30. MR 16,1014d Zbl 0065.30401

[Bruckman 1982] P. S. Bruckman, “Problem H-320”, Fibonacci Quart. 20:2 (1982), 186-187.

[Chu 1997] W. Chu, "Hypergeometric series and the Riemann zeta function", Acta Arith. 82:2 (1997), 103-118. MR 98m:11089 Zbl 0881.11066

[Connon 2007] D. F. Connon, "Elementary evaluations of some Euler sums", preprint, 2007. arXiv 0710.5360

[Connon 2008a] D. F. Connon, "Euler-Hurwitz series and non-linear Euler sums", preprint, 2008. arXiv 0803.1304

[Connon 2008b] D. F. Connon, "Some series and integrals involving the Riemann zeta function, binomial coefficients and the harmonic numbers, I", preprint, 2008. arXiv 0710.4022

[Connon 2008c] D. F. Connon, "Some series and integrals involving the Riemann zeta function, binomial coefficients and the harmonic numbers, IIa", preprint, 2008. arXiv 0710.4023

[Connon 2008d] D. F. Connon, "Some series and integrals involving the Riemann zeta function, binomial coefficients and the harmonic numbers, IIb", preprint, 2008. arXiv 0710.4024

[Connon 2008e] D. F. Connon, "Some series and integrals involving the Riemann zeta function, binomial coefficients and the harmonic numbers, III", preprint, 2008. arXiv 0710.4025

[Connon 2008f] D. F. Connon, "Some series and integrals involving the Riemann zeta function, binomial coefficients and the harmonic numbers, IV", preprint, 2008. arXiv 0710.4028

[Connon 2008g] D. F. Connon, "Some series and integrals involving the Riemann zeta function, binomial coefficients and the harmonic numbers, V", preprint, 2008. arXiv 0710.4047

[Connon 2008h] D. F. Connon, "Some series and integrals involving the Riemann zeta function, binomial coefficients and the harmonic numbers, VI", preprint, 2008. arXiv 0710.4032

[de Doelder 1991] P. J. de Doelder, "On some series containing $\psi(x)-\psi(y)$ and $(\psi(x)-\psi(y))^{2}$ for certain values of $x$ and $y$ ", J. Comput. Appl. Math. 37:1-3 (1991), 125-141. MR 92m:40002 Zbl 0782.33001

[Dumont 1981] D. Dumont, "Matrices d'Euler-Seidel”, Sémin. Lothar. Comb. 5:B05c (1981), 1-25. Zbl 0925.05025

[Euler 1776] L. Eulerus, "Meditationes circa singulare serierum genus", Novi Comm. Acad. Sci. Petropolitanae 20 (1776), 140-186. Reprinted on pp. 217-265 in his Opera Omnia, series prima, vol. 15 (Commentationes analyticae 2), Teubner, Berlin, 1927; the relevant passage starts on p. 228.

[Farnum and Tissier 1999] N. R. Farnum, "Problems and solutions", solution by A. Tissier of problem 10635, “Apery's constant”, Amer. Math. Monthly 106:10 (1999), 965-966. MR 1543578

[Flajolet and Salvy 1998] P. Flajolet and B. Salvy, "Euler sums and contour integral representations", Experiment. Math. 7:1 (1998), 15-35. MR 99c:11110 Zbl 0920.11061

[Gradshteyn and Ryzhik 2007] I. S. Gradshteyn and I. M. Ryzhik, Table of integrals, series, and products, 7th ed., Academic Press, Amsterdam, 2007. MR 2008g:00005 Zbl 1208.65001 
[Guillera and Sondow 2008] J. Guillera and J. Sondow, "Double integrals and infinite products for some classical constants via analytic continuations of Lerch's transcendent", Ramanujan J. 16:3 (2008), 247-270. MR 2009e:11239 Zbl 1216.11075

[Hoffman 1992] M. E. Hoffman, "Multiple harmonic series”, Pacific J. Math. 152:2 (1992), 275-290. MR 92i:11089 Zbl 0763.11037

[Klamkin and Steinberg 1952] M. S. Klamkin and R. Steinberg, "Advanced Problems and Solutions: Solutions: 4431", Amer. Math. Monthly 59:7 (1952), 471-472. MR 1528216

[Lewin 1981] L. Lewin, Polylogarithms and associated functions, North-Holland, New York, 1981. MR 83b:33019 Zbl 0465.33001

[Lewin 1991] L. Lewin (editor), Structural properties of polylogarithms, Mathematical Surveys and Monographs 37, Amer. Math. Soc., Providence, RI, 1991. MR 93b:11158 Zbl 0745.33009

[Li 2011] Z. Li, "On harmonic sums and alternating Euler sums", preprint, 2011. arXiv 1012.5192v3

[Mezó 2013] I. Mezô, "A $q$-Raabe formula and an integral of the fourth Jacobi theta function”, $J$. Number Theory 133:2 (2013), 692-704. MR 2994381 Zbl 06124189

[Mezô and Dil 2009] I. Mezô and A. Dil, "Euler-Seidel method for certain combinatorial numbers and a new characterization of Fibonacci sequence", Cent. Eur. J. Math. 7:2 (2009), 310-321. MR 2010c:11036 Zbl 1229.11043

[Seidel 1877] L. Seidel, "Über eine einfache Enstehung weise der Bernoullischen Zahlen und einiger verwandten Reihen”, Sitzungsberichte der Münch. Akad. Math. Phys. Classe 4 (1877), 157-187.

[Shen 1995] L.-C. Shen, "Remarks on some integrals and series involving the Stirling numbers and $\zeta(n) ”$, Trans. Amer. Math. Soc. 347:4 (1995), 1391-1399. MR 95g:11014 Zbl 0828.11044

[Sitaramachandra Rao 1987] R. Sitaramachandra Rao, "A formula of S. Ramanujan", J. Number Theory 25:1 (1987), 1-19. MR 88c:11048 Zbl 0606.10032

[Sofo and Hassani 2012] A. Sofo and M. Hassani, "Quadratic harmonic number sums", Appl. Math. E-Notes 12 (2012), 110-117. MR 2992944

[Ulanskii 2003] E. A. Ulanskii, "Identities for generalized polylogarithms", Mat. Zametki 73:4 (2003), 613-624. In Russian; translated in Math. Notes 73:4 (2003), 571-581. MR 2004f:11065 Zbl 1093.11049

[Wan 2012] J. Wan, "Some notes on weighted sum formulae for doubling zeta values", preprint, 2012. arXiv 1206.2424v1

Received August 23, 2013. Revised October 4, 2013.

ISTVÁN MEZŐ

DEPARTMENT OF MATHEMATICS

NANJING UNIVERSITY OF INFORMATION SCIENCE AND TECHNOLOGY

NANJING, 210044

CHINA

istvanmezo81@gmail.com 


\title{
PACIFIC JOURNAL OF MATHEMATICS
}

\author{
msp.org/pjm
}

Founded in 1951 by E. F. Beckenbach (1906-1982) and F. Wolf (1904-1989)

\section{EDITORS}

Don Blasius (Managing Editor)

Department of Mathematics

University of California

Los Angeles, CA 90095-1555

blasius@math.ucla.edu

\author{
Paul Balmer \\ Department of Mathematics \\ University of California \\ Los Angeles, CA 90095-1555 \\ balmer@math.ucla.edu \\ Robert Finn \\ Department of Mathematics \\ Stanford University \\ Stanford, CA 94305-2125 \\ finn@math.stanford.edu \\ Sorin Popa \\ Department of Mathematics \\ University of California \\ Los Angeles, CA 90095-1555 \\ popa@math.ucla.edu
}

\author{
Vyjayanthi Chari \\ Department of Mathematics \\ University of California \\ Riverside, CA 92521-0135 \\ chari@math.ucr.edu \\ Kefeng Liu \\ Department of Mathematics \\ University of California \\ Los Angeles, CA 90095-1555 \\ liu@math.ucla.edu \\ Jie Qing \\ Department of Mathematics \\ University of California \\ Santa Cruz, CA 95064 \\ qing@ cats.ucsc.edu
}

\section{PRODUCTION}

Silvio Levy, Scientific Editor, production@msp.org

\section{SUPPORTING INSTITUTIONS}

ACADEMIA SINICA, TAIPEI

CALIFORNIA INST. OF TECHNOLOGY

INST. DE MATEMÁTICA PURA E APLICADA

KEIO UNIVERSITY

MATH. SCIENCES RESEARCH INSTITUTE

NEW MEXICO STATE UNIV.

OREGON STATE UNIV.

\author{
STANFORD UNIVERSITY \\ UNIV. OF BRITISH COLUMBIA \\ UNIV. OF CALIFORNIA, BERKELEY \\ UNIV. OF CALIFORNIA, DAVIS \\ UNIV. OF CALIFORNIA, LOS ANGELES \\ UNIV. OF CALIFORNIA, RIVERSIDE \\ UNIV. OF CALIFORNIA, SAN DIEGO \\ UNIV. OF CALIF., SANTA BARBARA
}

\author{
Daryl Cooper \\ Department of Mathematics \\ University of California \\ Santa Barbara, CA 93106-3080 \\ cooper@math.ucsb.edu \\ Jiang-Hua Lu \\ Department of Mathematics \\ The University of Hong Kong \\ Pokfulam Rd., Hong Kong \\ jhlu@maths.hku.hk \\ Paul Yang \\ Department of Mathematics \\ Princeton University \\ Princeton NJ 08544-1000 \\ yang@math.princeton.edu
}

These supporting institutions contribute to the cost of publication of this Journal, but they are not owners or publishers and have no responsibility for its contents or policies.

See inside back cover or msp.org/pjm for submission instructions.

The subscription price for 2014 is US $\$ 410 /$ year for the electronic version, and \$535/year for print and electronic.

Subscriptions, requests for back issues and changes of subscribers address should be sent to Pacific Journal of Mathematics, P.O. Box 4163, Berkeley, CA 94704-0163, U.S.A. The Pacific Journal of Mathematics is indexed by Mathematical Reviews, Zentralblatt MATH, PASCAL CNRS Index, Referativnyi Zhurnal, Current Mathematical Publications and Web of Knowledge (Science Citation Index).

The Pacific Journal of Mathematics (ISSN 0030-8730) at the University of California, c/o Department of Mathematics, 798 Evans Hall \#3840, Berkeley, CA 94720-3840, is published twelve times a year. Periodical rate postage paid at Berkeley, CA 94704, and additional mailing offices. POSTMASTER: send address changes to Pacific Journal of Mathematics, P.O. Box 4163, Berkeley, CA 94704-0163.

PJM peer review and production are managed by EditFLOW ${ }^{\circledR}$ from Mathematical Sciences Publishers.

\section{PUBLISHED BY}

\section{mathematical sciences publishers \\ nonprofit scientific publishing}

http://msp.org/

(C) 2014 Mathematical Sciences Publishers 


\section{PACIFIC JOURNAL OF MATHEMATICS}

Volume $272 \quad$ No. $1 \quad$ November 2014

Nonconcordant links with homology cobordant zero-framed surgery $\quad 1$ manifolds

JAE CHOON CHA and MARK POWELL

Certain self-homotopy equivalences on wedge products of Moore spaces

Ho Won CHOI and KeE Young LeE

Modular transformations involving the Mordell integral in Ramanujan's lost notebook

Youn-SEO CHOI

The $D$-topology for diffeological spaces

J. DANiEl Christensen, Gordon SinNAMON and ENXIN Wu

On the Atkin polynomials

AHMAD EL-Guindy and Mourad E. H. ISMAIL

Evolving convex curves to constant-width ones by a perimeter-preserving flow

LAIYUAN GaO and Shengliang PAN

Hilbert series of certain jet schemes of determinantal varieties

SudhiR R. GHORPADE, Boyan Jonov and B. A. SeTHuraman

On a Liu-Yau type inequality for surfaces

Oussama Hijazi, Sebastián Montiel and Simon Raulot

Nonlinear Euler sums

ISTVÁN MEZŐ

Boundary limits for fractional Poisson $a$-extensions of $L^{p}$ boundary functions 227 in a cone

LEI QIAO and TAO ZHAO

Jacobi-Trudi determinants and characters of minimal affinizations

STEVEN V SAM

Normal families of holomorphic mappings into complex projective space concerning shared hyperplanes

LiU Yang, Caiyun Fang and Xuecheng Pang 